\title{
Alternative therapies in academic medical centers compromise evidence-based patient care
}

\author{
Donald M. Marcus
}

Department of Medicine, Baylor College of Medicine, Houston, Texas, USA.

D uring the past 20 years, integrative medicine centers that promote the use of alternative therapies were established in more than 70 medical and nursing schools. A survey of hospital websites and interviews with physicians, hospital administrators, and staff revealed that 15 research-intensive medical schools in the United States provide alternative therapies in their integrative medicine centers and affiliated hospitals (1). The treatments are claimed to provide benefits for a broad spectrum of diseases, including heart disease, cancer, digestive disorders, pain, autism, and autoimmune disorders. Duke Integrative Medicine offers acupuncture for treatment of 21 conditions, including rheumatoid arthritis, stroke, and essential hypertension. Johns Hopkins offers reiki therapy in which "the practitioner seeks to transmit Universal Life Energy to the client" (1). Hospital administrators state that they provide alternative therapies in response to consumer interest and that, even if those services don't generate profits, they build the hospitals' market share (1).

A recent article addressed the challenges of medical education in the era of alternative facts and the increasing use of social media to obtain information (2). It noted that medical educators should be "guiding our students past the barrage of misleading signals." Unfortunately, some of those misleading signals about the safety and efficacy of alternative therapies are coming from medical educators in integrative medicine centers.

\section{Alternative/integrative medicine}

There is no generally accepted definition of alternative therapies. The National Center for Complementary and Integrative Health (NCCIH), a unit of the NIH, defines them as "health care approaches developed outside of mainstream Western or conventional medicine" (3). That descriptive definition lacks a conceptual basis and is uninformative concerning the basic difference between alternative and conventional therapies. The defining characteristic of alternative therapies is that their health claims do not meet evidence-based standards, and many, such as naturopathy, homeopathy, and energy healing, are scientifically implausible. Moreover, integrative centers further confuse consumers by laying claim to therapies that are supported by sound evidence, such as exercise and mindfulness.

What is the rationale for alternative/ integrative medicine? Its advocates claim that evidence-based medicine is incapable of providing care for the whole person because advances in medical science have led to an algorithmic, impersonal model of medical practice (4). They also state that biomedicine overlooks the importance of a healthy lifestyle, preventive medicine, and mind-body interactions. The mission of integrative medicine is to compensate for these deficiencies by introducing safe, evidence-based alternative therapies into medical education and training.

It is remarkable that this misrepresentation of medical education and practice is a declaration that is not supported by any substantiating data, such as analyses of medical school curricula. Rather than being "algorithmic," evidence-based medicine integrates the best research evidence available, the experience of the physician, and the wishes of the patient in making decisions about treatment. It is a flexible and powerful approach to making clinical decisions (5). In a 2007 poll conducted by the British Medical Journal, evidence-based medicine was included in the

Conflict of interest: The author has declared that no conflict of interest exists.

15 most important advances in medicine since 1840 . The biopsychosocial model of medical training and the importance of a healthy lifestyle in preventing disease have been tenets of medical education for decades (6). Evaluation of the humanistic attributes of students, residents, and faculty has long been required by medical schools and by accrediting agencies and medical boards (7).

\section{Efficacy and safety of alternative therapies}

According to a National Health Statistics Survey, the most popular alternative therapies are chiropractic, acupuncture, and herbal remedies, which are misleadingly labeled dietary supplements. In 1994, US Congress enacted the Dietary Supplement and Health Education Act (DSHEA). Without any scientific basis, DSHEA arbitrarily classified herbal remedies and other medicinal products as dietary supplements, a category that previously included only vitamins and minerals. Plant extracts are used worldwide as medicines and not for their nutritional value. DSHEA prevents the FDA from effectively regulating dietary supplements and leads consumers to mistakenly believe that herbals are safe, like multivitamins. The consequence was a rapid increase in sales of herbal and other medicinal nonvitamin, nonmineral "supplements."

Claims for efficacy of alternative therapies are based primarily on clinical trials funded by commercial sources and professional organizations of alternative practitioners. Reviews of those trials concluded that they are uninterpretable because of their positive bias and poor quality $(8,9)$. An analysis of trials of glucosamine treatment of osteoarthritis revealed that all positive trials were funded by manufacturers, but no efficacy was noted in studies funded by independent, noncommercial sources. Likewise, rigorous trials supported by $\mathrm{NIH}$ and other noncommercial sources 
have failed to substantiate specific efficacy beyond a placebo effect for popular herbal supplements $(10,11)$, chiropractic manipulation for back pain (12), or acupuncture for knee osteoarthritis or back pain (13). Recent analyses of "all-natural" herbals revealed their lack of quality control and frequent adulteration by prescription drugs or analogs of hormones and stimulants $(10,11)$. In summary, expenditure of approximately $\$ 2.2$ billion by National Center for Complementary and Alternative Medicine (NCCAM)/NCCIH during fiscal years 1999-2017 for clinical trials produced no sound, consistent evidence for the efficacy of any alternative therapies. However, the grants lent academic credibility to integrative medicine.

Data about the frequency of adverse events caused by herbal "dietary supplements" are limited because DSHEA did not initially require manufacturers to report problems to the FDA. Based on data from Poison Control Centers, the FDA has estimated an annual incidence of 50,000 adverse events, many of which are serious (14). During 2004-2013, 23,005 visits to emergency departments were attributed to adverse events related to dietary supplements (15). Herbal or complementary nutritional products accounted for $65.9 \%$ of the events. A prospective study of hospital admissions for drug-induced acute hepatic inflammation found that $20 \%$ of cases were caused by dietary supplements (16). Other concerns are that herbal remedies may alter the activity of conventional medications and that alternative therapists without medical training may overlook a serious, treatable condition.

In theory, alternative treatments are used only to complement conventional treatment or by patients whose conditions have not responded to conventional therapy. However, some people choose alternative therapies as initial treatments, based on misleading claims for the safety and efficacy of "natural healing." Patients with four common cancers who chose alternative treatments as their sole initial therapy had a greater risk of death than matched controls who had conventional therapy (17).

\section{Education}

Integrative centers are also undermining evidence-based medical education. During 2000-2003, NIH provided educa- tional grants to health profession schools to integrate evidence-based complementary and alternative therapies into their curricula. A review of the integrative curricula revealed that they were outdated, were biased, and failed to meet evidence-based standards (18).

Academic integrative medicine centers have also received millions of dollars from private foundations whose donors believe in alternative therapies. The Osher Centers for Integrative Medicine provide funds to a number of centers, including those at UCSF, Harvard Medical School, Brigham and Women's Hospital, and Vanderbilt University School of Medicine. The University of California at Irvine received a gift of \$200 million from the Henry and Susan Samueli Foundation for a new building to house the Samueli College of Health Sciences (19). The College will emphasize integrative medicine throughout the curriculum. A portion of the gift will endow up to 15 chairs for faculty with "expertise in integrative health" (19). The Sidney Kimmel College of Medicine of Thomas Jefferson University recently created a new academic Department of Integrative Medicine and Nutritional Sciences (20). The initiative is supported by a $\$ 20$ million grant from the Marcus Foundation. The curriculum of the department will include "novel mechanisms of healing and emerging therapies." The role of philanthropy in creating new and expanded integrative medicine programs in medical schools raises concerns about the distortion of medical education.

Promotion of alternative therapies has also had economic consequences. Out-of-pocket expenditures in the United States for alternative products and services in 2012 were $\$ 30.2$ billion, which was approximately $24 \%$ of out-of-pocket expenditures for prescription drugs. Sales of nonvitamin, nonmineral supplements - mostly herbal remedies - were $\$ 12$ billion in 2012, approximately $39.7 \%$ of all expenditures for complementary health approaches.

\section{Conclusions}

Integrative medicine centers undermine evidence-based medical practice and education. They promote unsound and potentially hazardous therapies and provide flawed curricula to health care stu- dents and graduate physicians in training. Although supportive of patients' desire to play a meaningful role in managing their health care, physicians should adhere to standards of professionalism. The tenets of medical professionalism include a commitment to maintaining medical knowledge, a duty to uphold scientific standards, and a contract with society to provide expert advice on matters of health (21). Offering therapies that are not supported by evidence-based standards is a failure to adhere to professional tenets. It deceives patients and prevents them from making informed decisions about treatment options. Academic medical centers should be a source of sound advice for the public, instead of promoting unproven health practices.

Science is under unprecedented attack, at present. Biomedical scientists should speak up to support evidence-based health care. They should remind the public and members of US Congress that the remarkable advances in health care during the last 70 years were made possible by scientific research. Integrative medicine centers persist because of a lack of oversight by medical school faculty and administrators. Professional societies and individual scientists should urge medical schools to review the educational material in integrative medicine curricula and to establish ongoing oversight. Advocates for integrative medicine could argue that academic freedom gives them the right to express their beliefs. However, as educators and role models for learners, their primary responsibility is to uphold professional standards of integrity and science-based practice.

Address correspondence to: Donald M. Marcus, Department of Medicine, MS 285, Baylor College of Medicine, 1 Baylor Plaza, Houston, Texas 77030, USA. Phone: 713.798.6014; Email: dmarcus@bcm.edu.

1. Ross C, Blau M, Sheridan K. Medicine with a side of mysticism: Top hospitals promote unproven therapies. Stat News. https://www.statnews. com/2017/03/07/alternative-medicinehospitals-promote/. Published March 7, 2017. Accessed February 28, 2020.

2. Wenzel RP. Medical education in the era of alternative facts. $N$ Engl J Med 2017;377(7):607-609.

3. [No authors listed]. Complementary, Alternative, or Integrative Health: What's In a Name? NIH. https://nccih.nih.gov/sites/nccam.nih. 
gov/files/Whats_In_A_Name_06-16-2016.pdf. Accessed February 28, 2020.

4. Weil A. The significance of integrative medicine for the future of medical education. Am JMed. 2000;108(5):441-443.

5. Dickersin K, Straus SE, Bero LA. Evidence based medicine: increasing, not dictating, choice. $B M J$. 2007;334(Suppl 1):s10.

6. Engel GL. The need for a new medical model: a challenge for biomedicine. Science. 1977;196(4286):129-136.

7. [No authors listed]. Report 1: Learning objectives for medical student education-guidelines for medical schools: Guidelines for Medical Schools. Association of American Medical Colleges. https:// www.aamc.org/system/files/c/2/492708learningobjectivesformedicalstudenteducation. pdf. Accessed February 28, 2020.

8. Bausell RB. Snake Oil Science: the Truth About Complementary and Alternative Medicine. New York, NY: Oxford University Press; 2007.

9. Offit PA. Studying complementary and alternative therapies. JAMA. 2012;307(17):1803-1804.

10. Cohen PA. The supplement paradox: negligible benefits, robust consumption. JAMA. 2016;316(14):1453-1454.
11. Marcus DM. Dietary supplements: What's in a name? What's in the bottle? Drug Test Anal. 2016;8(3-4):410-412.

12. Cherkin DC, Sherman KJ, Deyo RA, Shekelle PG. A review of the evidence for the effectiveness, safety, and cost of acupuncture, massage therapy, and spinal manipulation for back pain. Ann Intern Med. 2003;138(11):898-906.

13. Madsen MV, Gøtzsche PC, Hróbjartsson A. Acupuncture treatment for pain: systematic review of randomised clinical trials with acupuncture, placebo acupuncture, and no acupuncture groups. BMJ. 2009;338:a3115.

14. [No authors listed]. Dietary Supplements: FDA Should Take Further Actions to Improve Oversight and Consumer Understanding. United States Government Accountability Office. https://www.gao.gov/products/gao-09-250. Published January 29, 2009. Accessed February 28, 2020.

15. Geller AI, et al. Emergency department visits for adverse events related to dietary supplements. N EnglJ Med. 2015;373(16):1531-1540.

16. Navarro VJ, Khan I, Björnsson E, Seeff LB, Serrano J, Hoofnagle JH. Liver injury from herbal and dietary supplements. Hepatology.
2017;65(1):363-373.

17. Johnson SB, Park HS, Gross CP, Yu JB. Use of alternative medicine for cancer and its impact on survival. J Natl Cancer Inst. 2018;110(1):121-124.

18. Marcus DM, McCullough L. An evaluation of the evidence in "evidence-based" integrative medicine programs. Acad Med.2009;84(9):1229-1234.

19. UCI receives $\$ 200$ million gift to name College of Health Sciences and launch major integrative health initiative. News release. UCI news. September 17, 2018. Accessed February 28, 2020. https://news.uci.edu/2017/09/18.

20. Thomas Jefferson University Launches Nation's First Academic Department of Integrative Medicine. News release. Jefferson News and Events. February 18, 2019. Accessed February 28, 2020. https://www.jefferson.edu/university/ news/2019/02/18/jefferson-launches-nationsfirst-academic-dept-integrative-medicine.html.

21. ABIM Foundation. American Board of Internal Medicine, ACP-ASIM Foundation. American College of Physicians-American Society of Internal Medicine, European Federation of Internal Medicine. Medical professionalism in the new millennium: a physician charter. Ann Intern Med. 2002;136(3):243-246. 